\title{
Dieselförderung ist ungesund
}

\author{
B. Aufdereggen ${ }^{a}$, R. Moll ${ }^{b}$
}

Dieser Aussage ist der Nationalrat in der Frühjahrssession 2003 gefolgt und hat die gesundheitlichen Bedenken der Ärztinnen und Ärzte für Umweltschutz ernst genommen. Zur Debatte stand die in einer Motion von der UREK Ständerat geforderte Senkung der Mineralölsteuer auf Dieseltreibstoff, welche der Nationalrat nun abgelehnt hat. Wesentlich war sicher auch die Tatsache, dass sich das BAG und die FMH gegen die Senkung ausgesprochen hatten.

Im Gegensatz zu Benzinmotoren stossen Dieselmotoren sehr viel mehr Feinpartikel aus. Feinpartikel (weitere Begriffe sind Feinstaub oder Schwebestaub) sind ein Gemisch von kleinsten Staubteilchen, die einen aerodynamischen Durchmesser von weniger als 10 Mikrometer aufweisen und deshalb auch PM10 (Particulate Matter) genannt werden. Tatsache ist, dass in der Schweiz schon jetzt die Jahresmittelwerte für PM10 ganzjährig und grossflächig über den Grenzwerten der Luftreinhalteverordnung liegen und der Tagesmittelwert häufig und zum Teil stark überschritten wird. Die gesundheitlichen Auswirkungen von Feinpartikeln sind bekannt und zahlreiche Studien belegen den Zusammenhang zwischen der Feinpartikelkonzentration und Atemwegserkrankungen, Herz-/ Kreislaufkrankheiten, Krebserkrankungen und vorzeitigen Todesfällen.

Die beiden grossen Schweizer Studien über Luftverschmutzung und Lungenkrankheiten (SAPALDIA [1] und SCARPOL [2]) zeigen, dass die Lungenfunktion Erwachsener mit zunehmender Feinpartikel- und Stickoxidbelastung schlechter wird und Atemwegsprobleme zunehmen. Je höher die Feinpartikelbelastung am Wohnort ist, desto häufiger kommen Infektionskrankheiten der Atemwege und ständiger Husten bei Kindern vor.

Der kurzfristige Anstieg des PM10-Durchschnitts um 100\% hatte bei Kindern eine Zunahme von Asthmanotfällen um 10\% zur Folge, dies zeigt eine Studie, die während zweier Jahre alle Kinder erfasste, die wegen Asthma notfallmässig ins grösste Spital von Belfast eingeliefert wurden [3]. Dass der Anstieg der Feinpartikel Asthmanotfälle bei Kindern auslösen kann, belegt eine weitere Studie aus Toronto [4]. Eine multizentrische Studie, die
Daten aus Spitälern in acht europäischen städtischen Gebieten (Barcelona, Birmingham, London, Mailand, den Niederlanden, Paris, Rom und Stockholm) analysierte, belegt eine Zunahme der Spitaleinweisungen wegen Herz-/Kreislaufkrankheiten bei kurzfristigem Anstieg des PM10Durchschnittswertes. Die Autoren schliessen aus ihren Resultaten, dass die Wirkung hauptsächlich auf Dieselabgase zurückzuführen sei [5]. Studien aus den Niederlanden und Boston zeigen, dass bei steigender Feinpartikelbelastung ein zunehmendes Risiko für einen Herzinfarkt besteht [6]. Die bisher umfassendste Kohortenstudie, in der in den USA über eine halbe Million Personen während mehr als 15 Jahren nachkontrolliert wurden, bestätigt nicht nur den Zusammenhang zwischen langfristig mittlerer Feinpartikelbelastung mit verminderter Lebenserwartung, sondern insbesondere auch die $\mathrm{Zu}-$ nahme der Lungenkrebshäufigkeit [7].

In dieser Situation muss es das Bestreben von Medizin, Politik und Behörden sein, die Luftbelastung mit Feinpartikeln zu senken. Die Ärztinnen und Ärzte für Umweltschutz haben in einem Brief an die Nationalrätinnen und Nationalräte die gesundheitlichen Argumente dargelegt und sie gebeten, die Motion zur Senkung der Mineralölsteuer auf Dieseltreibstoffen abzulehnen. Die Förderung von Diesel ist zudem aus Sicht des Klimaschutzes nicht angebracht, denn der Ausstoss der zusätzlichen Dieselrusspartikel wirkt klimaerwärmend [8] und hebt den Effekt der etwas geringeren $\mathrm{CO}_{2}$-Emissionen auf oder übertrifft ihn gar. Der Nationalrat ist den Argumenten der Ärzteschaft gefolgt und hat einen ersten Riegel gegen die Förderung von Dieseltreibstoff geschoben.

Unverständlich ist die gute Bewertung von Dieselfahrzeugen im Rahmen der neuen Energieetikette. Diese Bewertung ist lediglich aus der Optik des Energieverbrauchs entstanden und bezieht die Gefahren für Gesundheit und Klima nicht ein. Die Propagierung von Dieselfahrzeugen - insbesondere auch Personenwagen - ist solange fehl am Platz, als nicht alle mit Partikelfiltern und DeNox-Katalysatoren ausgerüstet sind. 


\section{Literatur}

1 Leuenberger P, Ackermann-Liebrich U, Kunzli N, Schindler C, Perruchoud AP. SAPALDIA: passé, présent et avenir. Schweiz Med Wochenschr 2000;130(8):291-7.

2 Braun-Fahrlander C, Vuille JC, Sennhauser FH, Neu U, Kunzle T, Grize L, et al. Respiratory health and long-term exposure to air pollutants in Swiss schoolchildren. Am J Respir Crit Care Med 1997; 155(3):1042-9.

3 Thompson AJ, Shields MD, Patterson CC. Acute asthma exacerbation and air pollutants in children living in Belfast. Arch Environ Health 2001;56(3):234-41.

4 Lin M, Chen Y, Burnett RT, Villeneuve PJ, Krewski D. The influence of ambient coarse particulate matter on asthma hospitalization in children. Environ Health Perspect 2002; 110(6):575-81.

5 Le Tertre A, Medina S, Samoli E, Forsberg B, Michelozzi P, Boumghar A, et al. Short-term effects of particulate air pollution on cardiovascular diseases in eight European cities. J Epidemiol Community Health 2002;56(10):773-9.
6 Peters A, Dockery DW, Muller JE, Mittleman MA. Increased particulate air pollution and the triggering of myocardial infarction. Circulation 2001;103(23):2810-5

7 Pope CA 3rd, Burnett RT, Thun MJ, Calle EE, Krewski D, Ito K, Thurston GD. Lung cancer, cardiopulmonary mortality and long-term exposure to fine particulate air pollution. J Am Med Assoc 2002;287(9):1132-41.

8 Jacobson MZ. Control of fossil-fuel particulate black carbon and organic matter, possibly the most effective method of slowing global warming. J Geophys Res 2002;107:16/1-16/22. http://www.stanford.edu/group/efmh/fossil/ fossil.pdf. 